How to cite: Bulzu, P.-A., Cristea, A., Buda, D.M., Banciu, H.L. (2019) Asgardarchaeota - A Novel Prokaryotic Group Discovered in Aquatic Sediments that Might Shed Light on the Origin and Early Evolution of Eukaryotes. 2019 "Air and Water - Components of the Environment" Conference Proceedings, Cluj-Napoca, Romania, p. 435446, DOI: 10.24193/AWC2019 43.

\title{
ASGARDARCHAEOTA - A NOVEL PROKARYOTIC GROUP DISCOVERED IN AQUATIC SEDIMENTS THAT MIGHT SHED LIGHT ON THE ORIGIN AND EARLY EVOLUTION OF EUKARYOTES
}

\author{
Paul-Adrian BULZU ${ }^{1 \S}$, Adorján CRISTEA ${ }^{1 \S}$, \\ Doriana M. BUDA ${ }^{1}$, Horia L. BANCIU ${ }^{I} \bowtie$ \\ DOI: 10.24193/AWC2019 43
}

\begin{abstract}
The origin of the eukaryotic cell and its shared evolutionary history with Archaea are among the hottest topics in modern biology. Recent improvements in cultureindependent genomics and phylogenomic analyses provided compelling evidence in support of the emergence of eukaryotes from within the Archaea. An important step towards revealing the identity and nature of the archaeal ancestor was made following metagenomics-based discovery of the Asgardarchaeota superphylum, a group of uncultivated archaea consisting of Loki-, Thor-, Heimdall- and Odinarchaeia. Their recognition as the closest extant relatives of the eukaryotes has reignited a decades-old debate regarding the topology of the Tree of Life. Moreover, genomic investigations of Asgardarchaeota revealed a plethora of "Eukaryote Signature Proteins" (ESPs), previously thought to be unique to eukaryotes, which may help shed light on the molecular events in early eukaryogenesis. In this work, we briefly review current knowledge about the geographical distribution, phylogeny, ESP content and metabolic capabilities of the highly diverse Asgardarchaeota in an attempt to picture the lifestyle and early evolution of eukaryotes.
\end{abstract}

Keywords. aquatic sediments, Asgardarchaeota, eukaryogenesis, eukaryote signature proteins

\section{ASGARDARCHAEOTA - NOVEL "MARVEL MICROBES"}

The emergence of the eukaryotes alongside their rapid diversification is widely regarded as one of the major events in the history of life (Adl et al., 2012; LópezGarcía and Moreira, 2015). Although recent insights place eukaryotes at the top of cellular evolution, stemming from an early interplay between an archaeal host (Eme et al., 2017) and an alphaproteobacterial endosymbiont (Martijn et al., 2018), questions persist on the exact identity and nature of the archaeal partner (ZarembaNiedzwiedzka et al., 2017; Da Cunha et al., 2018; Spang et al., 2018).

\footnotetext{
${ }^{1}$ Department of Molecular Biology and Biotechnology, Faculty of Biology and Geology, "BabeșBolyai" University, 5-7 Clinicilor Str., RO-400006, Cluj-Napoca, Romania. horia.banciu@ubbcluj.ro

$\S$ These authors contributed equally to this work.
} 
The origin of eukaryotes from within the Archaea, known as the "eocyte hypothesis" (Lake et al., 1984) and the two-domain Tree of Life model were first proposed in the late 1970s (Woese and Fox, 1977), at a time when (micro)biological knowledge was largely derived from cultured microbes (Bernard et al., 2018). Nevertheless, shortly after its stating, the eocyte hypothesis was obscured by the "three-domains Tree of Life" model (Woese and Fox, 1977) depicting Archaea and Eukarya as monophyletic sister lineages sharing a common ancestor. Recently, new insights brought by improved phylogenetic approaches and the advent of culture-independent methods such as metagenomics and single-cell genomics (Solden, Lloyd and Wrighton, 2016) have led to a resurgence of the eocyte hypothesis (Cox et al., 2008; Williams et al., 2013). This methodological leap that opened up a window towards the previously inaccessible "microbial dark matter" (Rinke et al., 2013) has also placed the eukaryotes in a new light by evidencing the mixed archaeo-bacterial structure of their genomes (Guy and Ettema, 2011). The metagenomics-based discovery of Lokiarchaeia and their phylogenomic placement as direct ancestors of the eukaryotes - a position previously held by members of the TACK superphylum (Guy and Ettema, 2011) - has fueled the already reignited debate about the topology of the Tree of Life (Zaremba-Niedzwiedzka et al., 2017; Da Cunha et al., 2018; Spang et al., 2018). This discovery was soon followed by three other novel candidate archaeal phyla (Odin -, Thor- and Heimdallarchaeia) that together with Lokiarchaeia comprise the recently defined Asgardarchaeota superphylum, or "Asgard archaea", named after the realm of gods in Norse mythology (Zaremba-Niedzwiedzka et al., 2017).

By December 2018, the inventory of Asgardarchaeota members consisted of 27 metagenome-assembled genomes (MAGs) deposited in the NCBI Genome database, 3 in the MG-RAST database, and 35 unpublished genomes reported in a recent preprint (Bulzu et al., 2018). The sixty-five MAGs include 10 Heimdallarchaeia, 26 Lokiarchaeia, 25 Thorarchaeia and one Odinarchaeia genome. In this work, we briefly review the current knowledge on the Asgard archaea, particularly the environments they inhabit, their phylogenetic positioning with respect to eukaryotes and other archaea, their peculiar genomic makeup and metabolic potential, all in an attempt to provide a unified view for future investigations into the subject of eukaryogenesis.

\section{GEOGRAPHICAL DISTRIBUTION AND ENVIRONMENT}

Following the discovery of Lokiarchaeia (Spang et al., 2015) in marine sediments from the Arctic Mid-Ocean Ridge, Asgard archaea were identified in a wide range of environments with markedly different biological and chemical parameters (Spang et al., 2015; Seitz et al., 2016; Dombrowski et al., 2017; Zaremba-Niedzwiedzka et al., 2017; Bulzu et al., 2018; Liu et al., 2018; Tully, Graham and Heidelberg, 2018).

Although evidence of Asgardarchaeota presence is frequently derived solely based on 16S rRNA genes or individual contigs (Zaremba-Niedzwiedzka et al., 
2017; Cai et al., 2018), the list of geographical sites that yielded more or less complete genomes is significantly shorter (Fig. 1).

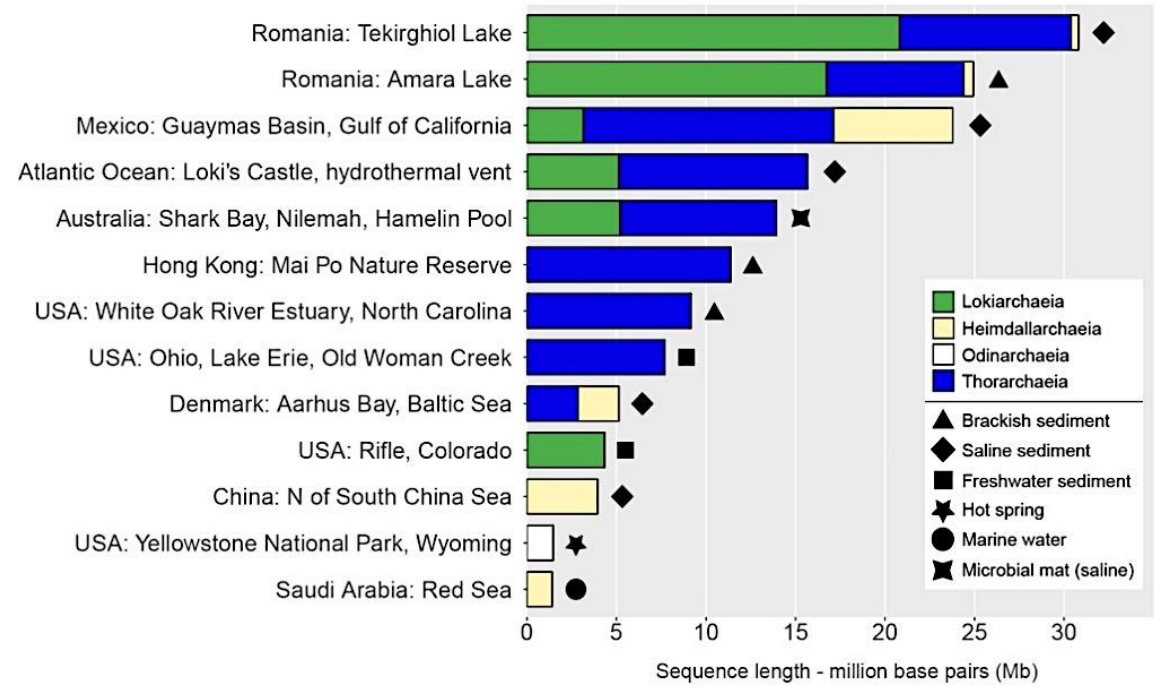

Fig. 1. The main geographical sites that have yielded Asgardarchaeota MAGs. Summed contig length is represented by horizontal bars, partitioned according to taxonomic affiliation. Taxonomy and environments are indicated in the legend.

Out of 13 sites, 10 are environments with low-to-high salinities (e.g., 1\% in Amara Lake versus 6\% in Tekirghiol Lake, both located in SE Romania), largely represented by aquatic sediments. Exceptions include the Hamelin Pool (Shark Bay, Australia) metagenome derived from hypersaline microbial mats (Suosaari et al., 2016) and the Red Sea marine water metagenome (Tully, Graham and Heidelberg, 2018). Additionally, two freshwater sediment metagenomes (from Old Woman Creek, Ohio, USA and Rifle, Colorado, USA) generated Thor- and Lokiarchaeia MAGs, respectively. Remarkably, the only available Odinarchaeia MAG was reconstructed from a hot spring $\left(\sim 70^{\circ} \mathrm{C}\right)$ sediment metagenome (Yellowstone National Park) while multiple individual contigs taxonomically affiliated to this phylum were assembled from sediment in the comparably hot $\left(63.3^{\circ} \mathrm{C}\right)$ Radiata Pool in New Zealand (Zaremba-Niedzwiedzka et al., 2017).

\section{ASGARDARCHAEOTA PHYLOGENOMICS}

The few available studies tackling the phylogenetic positioning of Asgardarchaeota lineages relative to eukaryotes as well as to other archaea derive their conclusions from analyses based on: 1) individual or concatenated 16S and 23S rRNA genes (Spang et al., 2015; Seitz et al., 2016; Zaremba-Niedzwiedzka et al., 2017; Bulzu et al., 2018; Liu et al., 2018), 2) concatenated ribosomal proteins conserved between archaea and eukaryotes (Spang et al., 2015; Zaremba- 
Niedzwiedzka et al., 2017; Bulzu et al., 2018), 3) concatenated universal marker genes (Da Cunha et al., 2017, 2018; Zaremba-Niedzwiedzka et al., 2017) and 4) individual marker genes - eukaryote signature proteins (ESPs) (Spang et al., 2015; Zaremba-Niedzwiedzka et al., 2017; Ak1l and Robinson, 2018; Bulzu et al., 2018) or other relevant markers such as elongation factor 2 (EF-2) (Narrowe et al., 2018).

Although most concatenated, as well as single marker gene phylogenies tend to recover the monophyletic relationship between Eukarya and Asgardarchaeota, analyses still fall short of reaching a consensus regarding the exact position of eukaryotes within the Asgard superphylum as well as that of individual Asgard phyla relative to each other (Zaremba-Niedzwiedzka et al., 2017; Bulzu et al., 2018). This situation can be mainly attributed to low taxon availability within the Asgardarchaeota and more generally to various challenges (i.e. sequence compositional heterogeneity, mutational saturation, heterotachy) that may cause phylogenetic artefacts (Delsuc, Brinkmann and Philippe, 2005; ZarembaNiedzwiedzka et al., 2017). Specifically, maximum likelihood trees constructed on rRNA genes depicted the eukaryotes emerging from within the Heimdallarchaeia clade (Zaremba-Niedzwiedzka et al., 2017; Bulzu et al., 2018) while bayesian inferences performed on SR4 recoded (Susko and Roger, 2007) ribosomal proteins failed to resolve the placement of the eukaryotic branch with respect to Loki- and Heimdallarchaeia. Nevertheless, these Bayesian approaches confidently support the monophyly between eukaryotes and Asgardarchaeota (Zaremba-Niedzwiedzka et al., 2017; Bulzu et al., 2018). Other interesting observations in the matter of eukaryogenesis also come from a recent study (Narrowe et al., 2018) addressing the evolutionary history of diphthamide biosynthesis and elongation factor 2 (EF-2) within archaea and the eukaryotes. The comparative analyses performed on EF-2 sequences have yielded additional phylogenetic, sequence and structural similarity evidence supporting Heimdallarchaeia as the closest extant archaeal relative of eukaryotes.

\section{EUKARYOTE SIGNATURE PROTEINS ARE ABUNDANT IN ASGARD ARCHAEA}

Study of Asgardarchaeota genomes revealed the largest number of genes coding for "eukaryote signature proteins" (ESPs) (Hartman and Fedorov, 2002) detected in any known archaeal lineage (Spang et al., 2015, 2018; ZarembaNiedzwiedzka et al., 2017). Identified ESP genes encode homologues of eukaryotic proteins acting in membrane maintenance and trafficking, endosomal sorting, ribosome structure, $\mathrm{N}$-glycosylation, protein ubiquitination as well as cytoskeleton structure and dynamics (e.g., profilin, actin, gelsolin, folliculin, etc.) (Spang et al., 2015; Zaremba-Niedzwiedzka et al., 2017; Bulzu et al., 2018; Liu et al., 2018).

Although there has been considerable debate (Da Cunha et al., 2018; Spang et al., 2018) whether these genes code for functional proteins, going as far as suggesting most ESPs could be the result of eukaryotic contamination in the metagenomes (Da Cunha et al., 2018), research efforts are currently being directed 
towards solving such concerns. First steps in this sense were made in a recent study (Ak1l and Robinson, 2018) that brought experimental evidence for a functional eukaryotic-like actin machinery in Asgard archaea while allegations of eukaryotic contamination (Da Cunha et al., 2018) were convincingly dismissed elsewhere (Spang et al., 2018). Yet, another study (Levasseur et al., 2017) argued that eukaryotic genes within the first Lokiarchaeia genome (Spang et al., 2015) might be the result of inter-domain "sequence exchange" (i.e., lateral gene transfer) while also questioning the validity of the Tree of Life model.

Notably, ESPs have been identified in the TACK group of archaeal lineages (Lindas et al., 2008; Ettema, Lindas and Bernander, 2011; Guy and Ettema, 2011; Yutin and Koonin, 2012; Williams et al., 2013; Koonin and Yutin, 2014) even before the discovery of the Asgard archaea. Comparative analyses between TACK and Asgardarchaeota indicate they share informational and ribosomal ESPs related to evolutionarily conserved functions while those involved in cellular structures and processes hitherto described only in eukaryotes are mostly specific to the latter (Fournier and Poole, 2018). Exceptions include the ESCRT-II protein, actin and tubulin homologues, which are present in both superphyla.

Taken together, the particularly large number of ESPs in Asgardarchaeota (Spang et al., 2015; Zaremba-Niedzwiedzka et al., 2017; Bulzu et al., 2018), their involvement in processes considered hallmark features for eukaryotes and their close phylogenetic relationship to eukaryotic homologues (Spang et al., 2015) further support the emergence of eukaryotes from within the Asgardarchaeota superphylum.

\section{METABOLICALLY VERSATILE MICROBES}

Apart from significant enthusiasm generated by phylogenomic evidence tracing the closest archaeal ancestor of eukaryotes within the Asgard archaea, great interest has been directed towards deciphering its cellular physiology. By doing so, it is assumed that knowledge regarding the fine cellular structure and metabolic capabilities of Asgardarchaeota will aid in testing competing hypotheses on the origin of eukaryotes (Koonin, 2015; Sousa et al., 2016; Bernard et al., 2018) thus ultimately providing valuable insights into the archaea-toeukarya transition. In lack of cultivable representatives, this scientific endeavor has relied exclusively upon state-of-the-art cultivation-independent metagenomics approaches.

As new Asgardarchaeota MAGs became available after the discovery of Lokiarchaeia in 2015 (Spang et al., 2015), studies reporting genome-scale metabolic reconstructions for members of this superphylum soon followed (Seitz et al., 2016; Sousa et al., 2016; Bulzu et al., 2018; Cai et al., 2018; Liu et al., 2018). Due to paucity of genomic data, less is known about Odinarchaeia metabolism, with only one available preprint article (Cai et al., 2018) attempting to tackle this subject. Overall, Asgard archaea are portrayed as mixotrophic microbes with markedly different phyla-level metabolic capabilities. In particular, genomic analyses revealed that Loki- and Thorarchaeia have anaerobic lifestyles (Bulzu et al., 2018; Cai et al., 2018; Liu et al., 2018), they both harbour the ancient Wood- 
Ljungdahl pathway (Borrel, Adam and Gribaldo, 2016) for inorganic carbon (CO2) fixation and perform acetogenesis, while Heimdallarchaeia display the metabolic blueprints for aerobiosis (Bulzu et al., 2018). Preferences towards the uptake of specific classes of organic compounds among Asgardarchaeota clades was reflected by genomic enrichment with genes encoding particular transporters (Bulzu et al., 2018; Liu et al., 2018). Thus, Lokiarchaeia showed a strong tendency towards the uptake of various monosaccharides which they may obtain by degrading cellulose, Thorarchaeia were enriched in peptide, glucarate and dicarboxylate transporters while $\mathrm{ABC}$-type transporters for phosphonates were specific for Heimdallarcheia. To some extent, all three phyla encode transporters for peptides/amino-acids uptake along with the enzymatic machinery required for their metabolization. As expected, substrate uptake preferences together with the ability to use molecular oxygen are mirrored within Asgardarchaeota central metabolism. In this sense, glycolysis is well represented in Lokiarchaeia, that appears endowed with sugar fermenting capabilities as well as in Heimdallarchaeia, where it shuttles intermediates into the tricarboxilyc acid cycle (TCA) thus fueling the electron transport chain (ETC). Surprisingly, the first key enzyme of the glycolytic pathway (i.e., glucokinase), as well as the TCA cycle, were lacking in Thorarchaeia (Bulzu et al., 2018; Liu et al., 2018). The complete TCA found in Lokiarchaeia MAGs shows the additional features required for anaplerotic $\mathrm{CO} 2$ fixation (i.e. achieved by the reverse TCA - rTCA). Additionally, the presence of type III-like RuBisCO genes within Loki- and Heimdallarchaeia MAGs indicates they are capable of $\mathrm{CO} 2$ fixation by linking nucleoside catabolism to glycolysis (Bulzu et al., 2018; Liu et al., 2018). Although incomplete, most of the pathway responsible for butane oxidation was identified in Thorarchaeia where this compound may serve as a source of energy (Cai et al., 2018; Liu et al., 2018).

A truly unexpected finding among aquatic sediment-dwelling archaea was the presence of multiple types of rhodopsin-encoding genes within Asgardarchaeota MAGs (Bulzu et al., 2018). This includes the novel schizorhodopsins (Bulzu et al., 2018) found in Loki-, Thor-, and Heimdallarchaeia, as well as heliorhodopsins (Pushkarev et al., 2018) and type-1 rhodopsins (Ruiz-Gonzalez and Marin, 2004), both found solely in the latter. The presence and role of light-harvesting rhodopsins in Asgardarchaeota is still unclear and will require further investigations.

Uniquely among Archaea, three Heimdallarchaeia MAGs (LC_2, LC_3, RS678) were found to harbour the complete pathway responsible for the aerobic degradation of tryptophan (i.e. the kynurenine pathway (Kurnasov et al. 2003)) (Bulzu et al., 2018). Together with the comparatively ubiquitous aspartate degradation pathway, also present in Heimdallarchaeia, kynurenine reactions produce the quinolinate required for de novo synthesis of nicotinamide adenine dinucleotide (NAD+) (Ternes and Schönknecht, 2014). Phylogenetic analyses (Bulzu et al., 2018) suggested that components of this pathway were acquired in the ancestor of Heimdallarchaeia by horizontal gene transfer (HGT) from bacteria.

Aside from providing further support for the aerobic metabolism of Heimdallarchaeia, the presence of the kynurenine pathway has major evolutionary 
implications since it is expected to have been present in the protoeukaryote ancestor (Ternes and Schönknecht, 2014). Taking this observation into account along with the phylogenomics and other aerobiosis-related evidence, it was suggested that the last archaeal ancestor of the eukaryotes was in fact a microaerophilic organism sharing a common ancestor with extant Heimdallarchaeia (Bulzu et al., 2018). Moreover, this view is supported by a recent high-scale time-calibrated phylogenomic reconstruction placing the moment of the archaeal-bacterial endosymbiosis that spawned the protoeukaryote ancestor after the Great Oxidation Event (Betts et al., 2018). This comes as an alternative view to the "hydrogen hypothesis" (Martin and Muller, 1998) that favors hydrogen-dependent and strictly anaerobic Lokiarchaeia (Sousa et al., 2016) as the archaeal host in the symbiosis that gave rise to the eukaryotes.

\section{CONCLUSIONS}

Taxonomy and abundance information derived from genomic as well as $16 \mathrm{~S}$ rRNA gene sequence data reveal that Asgard archaea are diverse microbes inhabiting various ecological niches all around the Earth.

Phylogenomic and phylogenetic analyses of conserved archaeo-eukaryotic genes provide evidence for the emergence of eukaryotes from within Asgardarchaeota thus supporting a two-domain Tree of Life.

The plethora of eukaryote signature proteins identified within Asgardarchaeota brings useful insights into the rise of eukaryotic cellular structures and eukaryotic-specific processes.

The available Asgardarchaeota genomic data provided valuable glimpses into their lifestyle and metabolism, eventually revealing divergent metabolic capabilities between phyla.

Two competing hypotheses for the origin of eukaryotes from within Asgardarchaeota are currently suggested: the "hydrogen hypothesis" and the "aerobicprotoeukaryotes" model, each presenting different mechanisms and environmental settings for this event. A better understanding of Asgard archaea diversity and metabolism will be critical for narrowing down among competing hypotheses regarding the emergence of the protoeukaryotic ancestor. Similarly, recovery of additional Odinarchaeia genomes will be crucial for making the first steps in describing the metabolic adaptations of these expectedly thermophilic archaea.

Progress towards unraveling the fine metabolic blueprints of Asgard archaea will largely depend on increased taxon sampling and sequencing, backed up by metagenomics, metatranscriptomics and hopefully by the isolation of these microbes.

\section{DATA AVAILABILITY}

Metagenomic datasets and assembled genomes used for making Figure 1 are available by their accession numbers in: NCBI (https://www.ncbi.nlm.nih.gov/) Amara and Tekirghiol Lakes, Romania, PRJNA483005; Guaymas Basin, Mexico, 
PRJNA362212; White Oak River Estuary, North Carolina, USA, PRJNA270657; Rifle, Colorado, USA, PRJNA288027; Aarhus Bay, Baltic Sea and Yellowstone National Park, USA, PRJNA319486; Loki's Castle, Atlantic Ocean, PRJNA259156; Red Sea, Saudi Arabia, PRJNA391943; South China Sea, PRJNA383916; Old Woman Creek, Ohio, USA, PRJNA406094; Mai Po Nature Reserve Marshes, Hong Kong, China, PRJNA360036; MG-RAST (https://www.mg-rast.org/) - Shark Bay, Australia, mgp81948. Figure 1 was made using the ggplot2 package (Wickham, 2016) implemented in the R statistical environment (RC Team, 2014).

\section{ACKNOWLEDGEMENTS}

This work was supported through funding granted by the Romanian National Authority for Scientific Research, CNCS - UEFISCDI, project number PN-III-P4ID-PCE-2016-0303.

\section{REFERENCES}

1. Adl, S.M., Simpson, A.G.B., Lane, C. E., Lukes, J., Bass, D., Bowser, S.S., Brown, M.W., Burki, F., Dunthorn, M., Hampl, V., Heiss, A., Hoppenrath, M., Lara, E., Le Gall, L., Lynn, D.H., McManus, H., Mitchell, E.A.D., Mozley-Stanridge, S.E., Parfrey, L.W., Pawlowski, J., Rueckert, S., Shadwick, L., Schoch, C.L., Smirnov, A., Spiegel, F.W. (2012), The revised classification of eukaryotes. J Eukaryot Microbiol. 59(5), 429-493. doi: 10.1111/j.1550-7408.2012.00644.x.

2. Ak11, C., Robinson, R.C. (2018), Genomes of Asgard archaea encode profilins that regulate actin, Nature, 562(7727), 439-443. doi: 10.1038/s41586-018-0548-6.

3. Bernard, G., Pathmanathan, J.S., Lannes, R., Lopez, P., Bapteste, E. (2018), Microbial dark matter investigations: How microbial studies transform biological knowledge and empirically sketch a logic of scientific discovery, Genome Biol Evol. 10(3), 707-715. http://dx.doi.org/10.1093/gbe/evy031.

4. Betts, H., Puttick, M., Clark, J., Williams, T., Donoghue, P., Pisani, D. (2018), Integrated genomic and fossil evidence illuminates life's early evolution and eukaryote origins, Nature Ecol Evol. 2, 1556-1562. https://doi.org/10.1038/s41559-018-0644-X.

5. Borrel, G., Adam, P. S., Gribaldo, S. (2016) Methanogenesis and the WoodLjungdahl Pathway: An Ancient, versatile, and fragile association, Genome Biol Evol. 8(6), 1706-1711. http://dx.doi.org/10.1093/gbe/evw114.

6. Bulzu, P.-A., Andrei, A.-S., Salcher, M.M., Mehrshad, M., Inoue, K., Kandori, H., Beja, O., Ghai, R., Banciu, H. (2018), The sunlit microoxic niche of the archaeal eukaryotic ancestor comes to light. bioRxiv, 385732. doi: 10.1101/385732.

7. Cai, M., Liu, Y., Zhou, Z., Yang, Y., Pan, J., Gu, J.-D., Li, M. (2018), Asgard archaea are diverse, ubiquitous, and transcriptionally active microbes, bioRxiv, 374165. doi: 10.1101/374165.

8. Cox, C.J., Foster, P.G., Hirt, R.P., Harris, S.R., Embley, T.M. (2008), The archaebacterial origin of eukaryotes. Proc Natl Acad Sci U S A. 105(51), 20356 20361. http://www.pnas.org/content/105/51/20356.abstract. 
9. Da Cunha, V., Gaia, M., Gadelle, D., Nasir, A., Forterre, P. (2017), Lokiarchaea are close relatives of Euryarchaeota, not bridging the gap between prokaryotes and eukaryotes. PLOS Genetics. 13(6), e1006810. https://doi.org/10.1371/journal.pgen.1006810.

10. Da Cunha, V., Gaia, M., Nasir, A., Forterre, P. (2018), Asgard archaea do not close the debate about the universal tree of life topology, PLOS Genetics. 14(3), e1007215. https://doi.org/10.1371/journal.pgen.1007215.

11. Delsuc, F., Brinkmann, H., Philippe, H. (2005), Phylogenomics and the reconstruction of the tree of life, Nat Rev Genet. 6, 361. https://doi.org/10.1038/nrg1603.

12. Dombrowski, N., Seitz, K. W., Teske, A. P., Baker, B. J. (2017), Genomic insights into potential interdependencies in microbial hydrocarbon and nutrient cycling in hydrothermal sediments. Microbiome. 5(1), 106. doi: 10.1186/s40168-017-0322-2.

13. Eme, L., Spang, A., Lombard, J., Stairs, C.W., Ettema, T.J.G. (2017), Archaea and the origin of eukaryotes. Nat Rev Microbiol. 15(12), 711-723. doi: 10.1038/nrmicro.2017.133.

14. Ettema, T.J.G., Lindas, A.-C., Bernander, R. (2011), An actin-based cytoskeleton in archaea. Mol Microbiol. 80(4), 1052-1061. doi: 10.1111/j.1365-2958.2011.07635.x.

15. Fournier, G.P., Poole, A.M. (2018), A briefly argued case that Asgard archaea are part of the Eukaryote Tree. Front Microbiol. 9, 1896. doi: 10.3389/fmicb.2018.01896.

16. Guy, L., Ettema, T.J.G. (2011), The archaeal "TACK" superphylum and the origin of eukaryotes. Trends Microbiol. 19(12), 580-587. doi: 10.1016/j.tim.2011.09.002.

17. Hartman, H., Fedorov, A. (2002), The origin of the eukaryotic cell: A genomic investigation. Proc Natl Acad Sci U.S.A., 99(3), 1420-1425. doi: 10.1073/pnas.032658599.

18. Koonin, E.V (2015), Archaeal ancestors of eukaryotes: not so elusive any more. BMC Biology. 13, 84. doi: 10.1186/s12915-015-0194-5.

19. Koonin, E.V., Yutin, N. (2014), The dispersed archaeal eukaryome and the complex archaeal ancestor of eukaryotes. Cold Spring Harbor perspectives in biology. Cold Spring Harbor Laboratory Press, 6(4), pp. a016188-a016188. doi: 10.1101/cshperspect.a016188.

20. Lake, J.A., Henderson, E., Oakes, M., Clark, M.W. (1984), Eocytes: a new ribosome structure indicates a kingdom with a close relationship to eukaryotes. Proc Natl Acad Sci U.S.A. 81(12), 3786-3790.

21. Levasseur, A., Merhej, V., Baptiste, E., Sharma, V., Pontarotti, P., Raoult, D. (2017), The rhizome of Lokiarchaeota illustrates the mosaicity of archaeal Genomes. Genome Biol Evol. 9(10), 2635-2639. doi: 10.1093/gbe/evx208.

22. Lindas, A.-C., Karlsson, E.A., Lindgren, M.T., Ettema, T.J.G. Bernander, R. (2008), A unique cell division machinery in the Archaea. Proc Natl Acad Sci U.S.A. 105(48), 18942-18946. doi: 10.1073/pnas.0809467105.

23. Liu, Y., Zhou, Z., Pan, J., Baker, B.J., Gu, J.-D., Li, M. (2018), Comparative genomic inference suggests mixotrophic lifestyle for Thorarchaeota. ISME J. 12(4), 1021-1031. doi: 10.1038/s41396-018-0060-x.

24. López-García, P., Moreira, D. (2015), Open questions on the origin of eukaryotes. Trends Ecol Evol. 30(11), 697-708. doi: 10.1016/j.tree.2015.09.005.

25. Martijn, J., Vosseberg, J., Guy, L., Offre, P., Ettema, T.J.G. (2018), Deep mitochondrial origin outside the sampled alphaproteobacteria. Nature. 557(7703), 101-105. doi: 10.1038/s41586-018-0059-5. 
26. Martin, W., Muller, M. (1998), The hydrogen hypothesis for the first eukaryote. Nature. 392(6671), 37-41. doi: 10.1038/32096.

27. Narrowe, A.B., Spang, A., Stairs, C.W., Caceres, E.F., Baker, B.J., Miller, C. S., Ettema, T.J.G. (2018), Complex evolutionary history of translation Elongation Factor 2 and diphthamide biosynthesis in Archaea and parabasalids, Genome Biol Evol. 10(9), 2380-2393. doi: 10.1093/gbe/evy154.

28. Pushkarev, A., Inoue, K., Larom, S., Flores-Uribe, J., Singh, M., Konno, M., Tomida, S., Ito, S., Nakamura, R., Tsunoda, S. P., Philosof, A., Sharon, I., Yutin, N., Koonin, E. V, Kandori, H., Beja, O. (2018), A distinct abundant group of microbial rhodopsins discovered using functional metagenomics. Nature. 558(7711), 595-599. doi: 10.1038/s41586-018-0225-9.

29. R Core Team (2014). R: A language and environment for statistical computing. R Foundation for Statistical Computing, Vienna, Austria. URL http://www.R-project.org/.

30. Rinke, C., Schwientek, P., Sczyrba, A., Ivanova, N.N., Anderson, I.J., Cheng, J.-F., Darling, A., Malfatti, S., Swan, B.K., Gies, E.A., Dodsworth, J. A., Hedlund, B.P., Tsiamis, G., Sievert, S.M., Liu, W.-T., Eisen, J.A., Hallam, S.J., Kyrpides, N.C., Stepanauskas, R., Rubin, E.M., Hugenholtz, P. Woyke, T. (2013), Insights into the phylogeny and coding potential of microbial dark matter. Nature. 499, 431-437. Available at: https://doi.org/10.1038/nature12352.

31. Ruiz-Gonzalez, M.X., Marin, I. (2004), New insights into the evolutionary history of type 1 rhodopsins. J Mol Evol. 58(3), 348-358. doi: 10.1007/s00239-003-2557-8.

32. Seitz, K.W., Lazar, C.S., Hinrichs, K.-U., Teske, A.P., Baker, B.J. (2016), Genomic reconstruction of a novel, deeply branched sediment archaeal phylum with pathways for acetogenesis and sulfur reduction. ISME J. 10(7), 1696-1705. doi: 10.1038/ismej.2015.233.

33. Solden, L., Lloyd, K., Wrighton, K. (2016), The bright side of microbial dark matter: lessons learned from the uncultivated majority. Curr Opin Microbiol. 31, 217-226. doi: https://doi.org/10.1016/j.mib.2016.04.020.

34. Sousa, F.L., Neukirchen, S., Allen, J.F., Lane, N., Martin, W.F. (2016), Lokiarchaeon is hydrogen dependent. Nat Microbiol, 1, 16034. doi: 10.1038/nmicrobiol.2016.34.

35. Spang, A., Eme, L., Saw, J.H., Caceres, E.F., Zaremba-Niedzwiedzka, K., Lombard, J., Guy, L., Ettema, T.J.G. (2018), Asgard archaea are the closest prokaryotic relatives of eukaryotes. PLOS Genetics. 14(3), e1007080. https://doi.org/10.1371/journal.pgen.1007080.

36. Spang, A., Saw, J. H., Jørgensen, S.L., Zaremba-Niedzwiedzka, K., Martijn, J., Lind, A.E., van Eijk, R., Schleper, C., Guy, L., Ettema, T.J.G. (2015), Complex archaea that bridge the gap between prokaryotes and eukaryotes. Nature. 521, 173179. http://dx.doi.org/10.1038/nature14447.

37. Suosaari, E.P., Reid, P.R., Araujo, T.A.A., Playford, P.E., Holley, D.K., McNamara, K.J., Eberli, G.P. (2016), Environmental pressures influencing living stromatolites in Hamelin Pool, Shark Bay, Western Australia. Palaios . 31(10), 483-496. doi: 10.2110/palo.2016.023.

38. Susko, E., Roger, A.J. (2007), On reduced amino acid alphabets for phylogenetic inference. Mol Biol Evol. 24(9), 2139-2150. doi: 10.1093/molbev/msm144.

39. Ternes, C.M., Schönknecht, G. (2014), Gene transfers shaped the evolution of de novo NAD (+) biosynthesis in eukaryotes', Genome Biol Evol. 6(9), 2335-2349. doi: $10.1093 /$ gbe/evu185. 
40. Tully, B.J., Graham, E.D., Heidelberg, J.F. (2018), The reconstruction of 2,631 draft metagenome-assembled genomes from the global oceans, Scientific Data. 5, 170203. https://doi.org/10.1038/sdata.2017.203.

41. Wickham, H. (2016) ggplot2: Elegant Graphics for Data Analysis. Springer-Verlag New York. http://ggplot2.org.

42. Williams, T.A., Foster, P.G., Cox, C.J., Embley, T.M. (2013), An archaeal origin of eukaryotes supports only two primary domains of life. Nature. 504, 231-236. https://doi.org/10.1038/nature12779.

43. Woese, C.R., Fox, G.E. (1977), Phylogenetic structure of the prokaryotic domain: the primary kingdoms. Proc Natl Acad Sci U.S.A. 74(11), 5088-5090. https://doi.org/10.1073/pnas.74.11.5088.

44. Yutin, N., Koonin, E.V (2012), Archaeal origin of tubulin. Biology Direct, 7, 10. doi: 10.1186/1745-6150-7-10.

45. Zaremba-Niedzwiedzka, K., Caceres, E.F., Saw, J.H., Backstrom, D., Juzokaite, L., Vancaester, E., Seitz, K.W., Anantharaman, K., Starnawski, P., Kjeldsen, K.U., Stott, M.B., Nunoura, T., Banfield, J.F., Schramm, A., Baker, B.J., Spang, A., Ettema, T.J.G. (2017), Asgard archaea illuminate the origin of eukaryotic cellular complexity. Nature. 541(7637), 353-358. doi: 10.1038/nature21031. 
